\title{
Average sex ratio and population maintenance cost
}

\author{
Eduardo Garibaldi \\ UNICAMP - Departamento de Matemática \\ 13083-859 Campinas - SP, Brasil \\ garibaldi@ime.unicamp.br
}

\author{
Marcelo Sobottka \\ UFSC - Departamento de Matemática \\ 88040-900 Florianópolis - SC, Brasil \\ sobottka@mtm.ufsc.br
}

\begin{abstract}
The ratio of males to females in a population is a meaningful characteristic of sexual species. The reason for this biological property to be available to the observers of nature seems to be a question never asked. Introducing the notion of historically adapted populations as global minimizers of maintenance cost functions, we propose a theoretical explanation for the reported stability of this feature. This mathematical formulation suggests that sex ratio could be considered as an indirect result shaped by the antagonism between the size of the population and the finiteness of resources.
\end{abstract}

Keywords: sex ratio, cost function, finite-resource environment, population dynamics.

Mathematical subject classification: 37N25, 92D15, 92D25.

This is a pre-copy-editing, author-produced PDF of an article accepted for publication in STAM Journal on Applied Mathematics, following peer review. The definitive publisher-authenticated version E. Garibaldi and M. Sobottka. Average sex ratio and population maintenance cost. SIAM Journal on Applied Mathematics (2011), 71, 1009-1025, doi:10.1137/100817310, is available online at: http://epubs.siam.org/doi/10.1137/100817310.

\section{Introduction}

"I formerly thought that when a tendency to produce the two sexes in equal numbers was advantageous to the species, it would follow from natural selection, but I now see that the whole problem is so intricate that it is safer to leave its solution for the future."

Charles Darwin in The descent of man [5].

It was reported [14] about five females for each male in Panamaniam colonies of spider Anelosimus eximius. This arachnid species has developed social groupings with overlap of generations, cooperation in care of young and reproductive division of labour. Male-biased sex ratio has been observed [1] in French populations of marmot Marmota marmota. This socially monogamous 
mammal is a cooperative breeding species and subordinate males participate in social thermoregulation during winter. In a territorial bird species, the Seychelles warbler Acrocephalus seychellensis, it was identified [11] a facultative adjustment of offspring sex ratio. The role of daughters as helpers in raising subsequent broods and the quality of a territory classified according to its size, the density of insect prey and the amount of foliage are factors that explain the sex ratio shift in offsprings, from mainly females on high-quality territories to mainly males on low-quality territories.

Sex-ratio studies form a fascinating topic in evolutionary biology, which underline the impact of natural selection on the allocation of resources to male and female progeny. Using a frequencydependent argument, Fisher provided [7] a theoretical explanation for the prevalence of near 1:1 sex ratio under natural selection. The effort to understand the stability of biased sex ratios has enabled the central theory to find successive and fruitful extensions. For instance, Hamilton's local mate competition hypothesis [9] was originally introduced to clarify how the interactions between siblings produce very female-biased sex ratios in parasitic wasps.

It has been very useful in sex-ratio theory the point of view which consists in describing collective phenomena from the actions and expectations of individuals. Charnov's book on sex allocation [4] is an extremely successful illustration of this tendency. By considering non-linear and unequal returns from parental investment in sons and daughters, Charnov has developed a nice mathematical formulation, able not only to conceptually explain cases of both Fisherian and non-Fisherian sex ratios, but also to provide predictions to be tested in experiments. Another example of a fundamental contribution from the philosophical approach based on methodological individualism is the so-called Triver-Willard hypothesis [13], which suggests that natural selection should favor parental ability to adjust the sex ratio of their offspring in response to environmental conditions.

The focus on individual behavior leads to the important discussion about selection criteria for reproductive strategies. Nowadays questions arising from parent-offspring conflict, parental investment, sibling antagonism and mate choice may be mathematically addressed by evolutionary game dynamics (see, for instance, [10]).

We will adopt a different point of view, which consists mostly in a global perspective by proposing a population-based optimization model. As any general model, this mathematical formulation will have mainly heuristic purposes. Focusing on the entire population as a dynamical agent without directly paying attention to specific biological parameters, the consideration of an implicit maintenance cost function will give qualitative insights for a common biological feature: an observable sex ratio. As a matter of fact, our main result will argue in favor of the hypothesis that the very possibility of a sex ratio being recognized in the nature may reflect a balance between the size of the population and the finiteness of resources.

The mathematical techniques developed here have foundations in common with the variational theory applied to the study of ground-states of generalized Frenkel-Kontorova models on a onedimensional crystal (see, for example, [2, 3]). Actually, statistical physics methods have been already successfully exploited in evolutionary games on graphs, specially when social networks are seen as the result of individual interactions governed by some kind of interdependency, such as sexual relationships (see, for example, [12]).

In order to be more concrete, suppose we periodically census the size of each gender in some biological population. Let $\left(\begin{array}{l}x_{i} \\ y_{i}\end{array}\right) \in \mathbb{N}^{2}$ be the $(i+1)^{t h}$ census, where $x_{i}$ and $y_{i}$ indicate the number of females and males, respectively. An infinite list $\omega=\left(\left(\begin{array}{l}x_{0} \\ y_{0}\end{array}\right),\left(\begin{array}{l}x_{1} \\ y_{1}\end{array}\right),\left(\begin{array}{l}x_{2} \\ y_{2}\end{array}\right), \ldots\right)$ can be viewed as a possible 
(yet maybe unlikely) historical record of each gender of a particular population. Obviously, not all $\omega$ has a biological meaning: this could be the case, for instance, of $\omega=\left(\left(\begin{array}{l}1 \\ 0\end{array}\right),\left(\begin{array}{l}0 \\ 0\end{array}\right),\left(\begin{array}{l}0 \\ 0\end{array}\right),\left(\begin{array}{c}0 \\ 99\end{array}\right),\left(\begin{array}{c}99 \\ 0\end{array}\right), \ldots\right)$. Hence one evidently needs some criteria to select among all registers those which may indeed represent a possible history of some population. This can be obtained by considering a function which associates some maintenance cost for any finite register of a population history. Such a cost function shall necessarily capture chief features 1 of the biological population to be modeled.

A cost function leads us to the notion of historically adapted populations, which intuitively correspond to those populations more efficient in the use of available resources. The concept of "historically adapted population" shall not be understood as "survival of the fittest". In fact, we are not focusing on competition either between species or among individuals, but only looking for the optimal rates for each gender in populations under certain environmental conditions. In particular, we neither claim that actual populations are historically adapted nor try to explain biological mechanisms which could lead a population to be historically adapted. Even so, the mathematical proof of a kind of abundance of historically adapted populations with an identifiable sex ratio might insinuate why sex-ratio random variations in a given population seem to be a very rare phenomenon in nature.

The paper is organized as follows. In section 2, we present the mathematical model we shall study. In section 3, we introduce the notion of historically adapted populations and show some of their properties. In section 4 , we present arguments for the existence of an asymptotic average sex ratio for historically adapted populations. Concluding remarks are discussed in section 5. In appendix A, one can find the mathematical proofs of the results used along the paper.

\section{The model}

In this section, we shall present a mathematical formulation to model two-sex populations in a finite-resource environment. Denote then the set of all nonnegative integers by $\mathbb{N}$. Define

$$
\Omega:=\left(\mathbb{N}^{2}\right)^{\mathbb{N}}:=\left\{\left(\begin{array}{l}
x_{i} \\
y_{i}
\end{array}\right)_{i \in \mathbb{N}}: x_{i}, y_{i} \in \mathbb{N}, \forall i \in \mathbb{N}\right\} .
$$

The elements of $\Omega$ will be called the (possible) histories for the population. Each history $\omega \in \Omega$ can be interpreted as a list of consecutive censuses of female and male populations. Given $\omega=$ $\left(\begin{array}{l}x_{i} \\ y_{i}\end{array}\right)_{i \in \mathbb{N}} \in \Omega$ and $m, n \in \mathbb{N}$ with $m \leq n$, we set $\omega[m]:=\left(\begin{array}{l}x_{m} \\ y_{m}\end{array}\right)$ and $\omega[m, n]:=\left(\begin{array}{l}x_{i} \\ y_{i}\end{array}\right)_{m \leq i \leq n}$, which are the restrictions of the infinite history $\omega$ to the moment $m$ and to the finite history from the moment $m$ until the moment $n$, respectively.

We will now define a class of cost functions which shall be used to select those censuses that may in fact be realized. First of all, we would like to emphasize that, although we will explicitly express only the dependence on population sizes, a maintenance cost function must depend on several biological and physical variables. We will just omit this multiple dependence in our analysis. Thus, let $C: \mathbb{N}^{2} \times \mathbb{N}^{2} \rightarrow \mathbb{R}$ be a function bounded from below, which means

$$
\inf _{\left(\left(\begin{array}{l}
x \\
y
\end{array}\right),\left(\begin{array}{l}
\bar{x} \\
\bar{y}
\end{array}\right)\right) \in \mathbb{N}^{2} \times \mathbb{N}^{2}} C\left(\left(\begin{array}{l}
x \\
y
\end{array}\right),\left(\begin{array}{l}
\bar{x} \\
\bar{y}
\end{array}\right)\right)>-\infty .
$$

\footnotetext{
${ }^{1}$ In particular, it must assign a high cost to finite population histories which should be unlikely.
} 
The value $C\left(\left(\begin{array}{l}x \\ y\end{array}\right),\left(\begin{array}{l}\bar{x} \\ \bar{y}\end{array}\right)\right)$ shall be interpreted as the maintenance cost to have a population with $x$ females and $y$ males, followed by a population with $\bar{x}$ females and $\bar{y}$ males. In particular, we are assuming that the maintenance cost takes into account only two successive population censuses. This assumption is made for simplicity and can be justified by observing that this model captures the main features of the general case, when the maintenance cost is a function of a finite number of consecutive censused-population sizes (see section [5).

In a finite-resource environment, it is reasonable to assume that, uniformly and independently of the initial population size, the cost to generate and maintain a new population tends to infinity as its size increases. In mathematical terms, the latter hypothesis can be expressed as follows

$$
\lim _{\bar{x}+\bar{y} \rightarrow+\infty} \inf _{\left(\begin{array}{l}
x \\
y
\end{array}\right) \in \mathbb{N}^{2}} C\left(\left(\begin{array}{l}
x \\
y
\end{array}\right),\left(\begin{array}{l}
\bar{x} \\
\bar{y}
\end{array}\right)\right)=+\infty .
$$

On the other hand, it is also reasonable to suppose that the population maintenance cost is, in some sense, more affected by the current population than by the former one. Roughly speaking, such an assumption means that, although the cost for a small initial population generating a very numerous new one may be high, the maintenance of a numerous population has a high cost by itself, independently of its previous size. Therefore, we shall assume that there exists a constant $\mathfrak{K}_{C}>0$ such that the cost of having $\left(\begin{array}{l}\bar{x} \\ \bar{y}\end{array}\right)$ in any census does not vary more than $\mathfrak{K}_{C}$ as a function of the possible values for the former population, or more precisely, we assume

$$
\mathfrak{K}_{C}:=\sup _{\left(\begin{array}{l}
\bar{x} \\
\bar{y}
\end{array}\right) \in \mathbb{N}^{2}}\left[\sup _{\left(\begin{array}{l}
x \\
y
\end{array}\right) \in \mathbb{N}^{2}} C\left(\left(\begin{array}{l}
x \\
y
\end{array}\right),\left(\begin{array}{l}
\bar{x} \\
\bar{y}
\end{array}\right)\right)-\inf _{\left(\begin{array}{l}
x \\
y
\end{array}\right) \in \mathbb{N}^{2}} C\left(\left(\begin{array}{l}
x \\
y
\end{array}\right),\left(\begin{array}{l}
\bar{x} \\
\bar{y}
\end{array}\right)\right)\right]<+\infty .
$$

\section{Historically adapted populations}

The intuitive idea is that a historically adapted population should minimize the maintenance cost along the time. Although, in most of the cases, there is no meaning in talking about a minimum cost for infinite histories, the idea of histories minimizing the cost along the time will be useful. As a matter of fact, this heuristic motivation will allow to highlight a central functional equation that will lead us to a rigorous definition of historically adapted populations.

\subsection{Heuristic motivation}

In order to explore heuristically a definition of historically adapted populations, consider that the function $C$ is nonnegative 2 . Note that the total maintenance cost of a population history $\bar{\omega}=\left(\begin{array}{l}\bar{x}_{i} \\ \bar{y}_{i}\end{array}\right)_{i \in \mathbb{N}}$ is given by $\sum_{k \geq 1} C\left(\left(\begin{array}{l}\bar{x}_{k-1} \\ \bar{y}_{k-1}\end{array}\right),\left(\begin{array}{l}\bar{x}_{k} \\ \bar{y}_{k}\end{array}\right)\right)$, which may clearly diverge. Assume for now that there exists a history with finite total maintenance cost (that is, for which the series converges). Thus, the smallest total cost for some history beginning from a given initial population $\left(\begin{array}{l}x_{0} \\ y_{0}\end{array}\right)$ is just

$$
u\left(\begin{array}{l}
x_{0} \\
y_{0}
\end{array}\right):=\inf _{\left(\left(\begin{array}{l}
x_{1} \\
y_{1}
\end{array}\right),\left(\begin{array}{l}
x_{2} \\
y_{2}
\end{array}\right), \ldots\right)}\left[\sum_{k \geq 1} C\left(\left(\begin{array}{l}
x_{k-1} \\
y_{k-1}
\end{array}\right),\left(\begin{array}{l}
x_{k} \\
y_{k}
\end{array}\right)\right)\right] .
$$

\footnotetext{
${ }^{2}$ Mathematically there is no loss of generality with such an assumption, since $C$ is bounded from below.
} 
Since we are assuming that the total cost is finite for some history, then $u\left(\begin{array}{l}x_{0} \\ y_{0}\end{array}\right) \in \mathbb{R}$ for any $\left(\begin{array}{l}x_{0} \\ y_{0}\end{array}\right) \in \mathbb{N}^{2}$. Moreover, as the cost function $C$ is supposed to be nonnegative, obviously $u \geq 0$ everywhere. Now, note that the above equation can be rewritten as

$$
\begin{aligned}
u\left(\begin{array}{l}
x_{0} \\
y_{0}
\end{array}\right) & =\inf _{\left(\begin{array}{l}
x_{1} \\
y_{1}
\end{array}\right)} \inf _{\left(\left(\begin{array}{l}
x_{2} \\
y_{2}
\end{array}\right),\left(\begin{array}{l}
x_{3} \\
y_{3}
\end{array}\right), \ldots\right)}\left[C\left(\left(\begin{array}{l}
x_{0} \\
y_{0}
\end{array}\right),\left(\begin{array}{l}
x_{1} \\
y_{1}
\end{array}\right)\right)+\sum_{k \geq 2} C\left(\left(\begin{array}{l}
x_{k-1} \\
y_{k-1}
\end{array}\right),\left(\begin{array}{l}
x_{k} \\
y_{k}
\end{array}\right)\right)\right] \\
& =\inf _{\left(\begin{array}{c}
x_{1} \\
y_{1}
\end{array}\right)}\left[C\left(\left(\begin{array}{l}
x_{0} \\
y_{0}
\end{array}\right),\left(\begin{array}{l}
x_{1} \\
y_{1}
\end{array}\right)\right)+\underset{\left.\left(\begin{array}{l}
x_{2} \\
y_{2}
\end{array}\right),\left(\begin{array}{c}
x_{3} \\
y_{3}
\end{array}\right), \ldots\right)}{\inf } \sum_{k \geq 2} C\left(\left(\begin{array}{l}
x_{k-1} \\
y_{k-1}
\end{array}\right),\left(\begin{array}{l}
x_{k} \\
y_{k}
\end{array}\right)\right)\right] \\
& =\inf _{\left(\begin{array}{c}
x_{1} \\
y_{1}
\end{array}\right)}\left[C\left(\left(\begin{array}{l}
x_{0} \\
y_{0}
\end{array}\right),\left(\begin{array}{l}
x_{1} \\
y_{1}
\end{array}\right)\right)+u\left(\begin{array}{l}
x_{1} \\
y_{1}
\end{array}\right)\right] .
\end{aligned}
$$

Hypothesis (2) implies that the infimum in the above equation is actually a minimum. Therefore, for each $\left(\begin{array}{l}x_{0} \\ y_{0}\end{array}\right) \in \mathbb{N}^{2}$, there exists $\left(\begin{array}{l}x_{1} \\ y_{1}\end{array}\right) \in \mathbb{N}^{2}$ such that

$$
u\left(\begin{array}{l}
x_{0} \\
y_{0}
\end{array}\right)=C\left(\left(\begin{array}{l}
x_{0} \\
y_{0}
\end{array}\right),\left(\begin{array}{l}
x_{1} \\
y_{1}
\end{array}\right)\right)+u\left(\begin{array}{l}
x_{1} \\
y_{1}
\end{array}\right) .
$$

One has now a recursive way to construct an interesting history. Indeed, given an initial population $\left(\begin{array}{l}x_{0} \\ y_{0}\end{array}\right)$, we find $\left(\begin{array}{l}x_{1} \\ y_{1}\end{array}\right)$ which satisfies ([6) and, inductively, from the population $\left(\begin{array}{l}x_{i-1} \\ y_{i-1}\end{array}\right)$ at the moment $i-1$, we obtain a population $\left(\begin{array}{l}x_{i} \\ y_{i}\end{array}\right)$ at the subsequent moment such that $u\left(\begin{array}{l}x_{i-1} \\ y_{i-1}\end{array}\right)=$ $C\left(\left(\begin{array}{l}x_{i-1} \\ y_{i-1}\end{array}\right),\left(\begin{array}{l}x_{i} \\ y_{i}\end{array}\right)\right)+u\left(\begin{array}{l}x_{i} \\ y_{i}\end{array}\right)$. Since its total maintenance cost is equal to the smallest one we could expect for some history beginning from $\left(\begin{array}{l}x_{0} \\ y_{0}\end{array}\right)$, the history $\omega=\left(\begin{array}{l}x_{i} \\ y_{i}\end{array}\right)_{i \in \mathbb{N}}$ constructed by the above procedure will be called an adapted history. We remark that there is not necessarily uniqueness and there might exist infinitely many adapted histories starting from a given initial population.

\subsection{Rigorous definition}

The existence of histories with finite total maintenance cost is a very strong demand made for our heuristic definition of historically adapted populations. Besides being a tremendous restriction for the model, such an assumption implies counterintuitively that the maintenance cost of these populations vanishes as time goes by. Anyway, it is not totally naive the observation that an adapted history should be one for which in some sense $C\left(\left(\begin{array}{l}x_{k-1} \\ y_{k-1}\end{array}\right),\left(\begin{array}{l}x_{k} \\ y_{k}\end{array}\right)\right)$ goes to the infimum of the cost function $C$ as $k$ tends to $+\infty$. More important, the previous heuristic discussion leads us to propose a general definition of adapted histories, which extends the intuitive idea of global minimizing histories for situations where the notion of finite total maintenance cost has no meaning.

It is straightforward that whenever a function $u: \mathbb{N}^{2} \rightarrow \mathbb{R}$ is bounded from below and verifies an equation like (5), we can use it to construct adapted histories. If the maintenance cost function $C$ satisfies hypotheses (11), (2) and (3), then one can show that there exist a bounded function ${ }^{3}$

\footnotetext{
${ }^{3}$ Such a function is a fixed point for a kind of Lax-Oleinik operator (see Definition 2 Theorem 3 and Remark 4 in appendix A).
} 
$u: \mathbb{N}^{2} \rightarrow \mathbb{R}$ and a real constant $\gamma$ (both depending on the function $C$ ) such that

$$
u\left(\begin{array}{l}
x \\
y
\end{array}\right)=\inf _{\left(\begin{array}{c}
\bar{x} \\
\bar{y}
\end{array}\right) \in \mathbb{N}^{2}}\left[C\left(\left(\begin{array}{l}
x \\
y
\end{array}\right),\left(\begin{array}{l}
\bar{x} \\
\bar{y}
\end{array}\right)\right)+u\left(\begin{array}{l}
\bar{x} \\
\bar{y}
\end{array}\right)\right]-\gamma, \quad \text { for all }\left(\begin{array}{l}
x \\
y
\end{array}\right) \in \mathbb{N}^{2} .
$$

Since $C$ satisfies (2) and $u$ is bounded, we can again deduce that for each $\left(\begin{array}{l}x_{0} \\ y_{0}\end{array}\right)$ there exists $\left(\begin{array}{l}x_{1} \\ y_{1}\end{array}\right)$ such that $u\left(\begin{array}{l}x_{0} \\ y_{0}\end{array}\right)=C\left(\left(\begin{array}{l}x_{0} \\ y_{0}\end{array}\right),\left(\begin{array}{l}x_{1} \\ y_{1}\end{array}\right)\right)+u\left(\begin{array}{l}x_{1} \\ y_{1}\end{array}\right)-\gamma$. Hence, given any initial population $\left(\begin{array}{l}x_{0} \\ y_{0}\end{array}\right)$, we define in a recursive way adapted histories starting from $\left(\begin{array}{l}x_{0} \\ y_{0}\end{array}\right)$ as we have made in section 3.1 .

Definition 1. Let $u: \mathbb{N}^{2} \rightarrow \mathbb{R}$ be a bounded function satisfying the functional equation (17) for some constant $\gamma$. Then $\omega=\left(\begin{array}{l}x_{i} \\ y_{i}\end{array}\right)_{i \in \mathbb{N}}$ is said to be an adapted history for the maintenance cost $C$ if

$$
u\left(\begin{array}{l}
x_{i-1} \\
y_{i-1}
\end{array}\right)=C\left(\left(\begin{array}{l}
x_{i-1} \\
y_{i-1}
\end{array}\right),\left(\begin{array}{l}
x_{i} \\
y_{i}
\end{array}\right)\right)+u\left(\begin{array}{l}
x_{i} \\
y_{i}
\end{array}\right)-\gamma, \quad \text { for all } i \geq 1 .
$$

We remark that in this context the quantity $u\left(\begin{array}{l}x_{0} \\ y_{0}\end{array}\right)$ is not necessarily given by the expression (44) and then it cannot be interpreted as the smallest total cost we would expect for any history starting from $\left(\begin{array}{l}x_{0} \\ y_{0}\end{array}\right)$. Anyway, if $\omega=\left(\begin{array}{l}x_{i} \\ y_{i}\end{array}\right)_{i \in \mathbb{N}}$ is an adapted history, then it is easy to see that its average maintenance cost tends to $\gamma$ as time goes by, or in mathematical terms

$$
\lim _{n \rightarrow \infty} \frac{1}{n} \sum_{k=1}^{n} C\left(\left(\begin{array}{l}
x_{k-1} \\
y_{k-1}
\end{array}\right),\left(\begin{array}{l}
x_{k} \\
y_{k}
\end{array}\right)\right)=\gamma
$$

Therefore, it follows from the functional equation (17) that $\gamma$ can be interpreted as the minimum asymptotic average maintenance cost we can expect for arbitrary histories, and this minimum value $\gamma$ is necessarily attained by any adapted history. Thus, even without uniqueness of adapted histories, we have uniqueness of the quantity $\gamma$ (see Remark 4 in appendix A).

If $\omega=\left(\begin{array}{l}x_{i} \\ y_{i}\end{array}\right)_{i \in \mathbb{N}}$ is an adapted history, we can recover the global minimizing property, since any finite history $\omega[m, n]=\left(\begin{array}{l}x_{i} \\ y_{i}\end{array}\right)_{m \leq i \leq n}$ minimizes the maintenance cost among all finite histories with the same initial and final population 4 . More precisely, for all $m, n \in \mathbb{N}$ with $m<n$ and for any other population history $\bar{\omega}=\left(\begin{array}{l}\bar{x}_{i} \\ \bar{y}_{i}\end{array}\right)_{i \in \mathbb{N}} \in \Omega$ verifying $\omega[m]=\bar{\omega}[m]$ and $\omega[n]=\bar{\omega}[n]$, it follows that

$$
\begin{aligned}
& \sum_{k=m+1}^{n} C\left(\left(\begin{array}{l}
x_{k-1} \\
y_{k-1}
\end{array}\right),\left(\begin{array}{l}
x_{k} \\
y_{k}
\end{array}\right)\right)={ }_{(1)} \sum_{k=m+1}^{n}\left[u\left(\begin{array}{l}
x_{k-1} \\
y_{k-1}
\end{array}\right)-u\left(\begin{array}{l}
x_{k} \\
y_{k}
\end{array}\right)+\gamma\right] \\
& ={ }_{(2)} u\left(\begin{array}{l}
x_{m} \\
y_{m}
\end{array}\right)-u\left(\begin{array}{l}
x_{n} \\
y_{n}
\end{array}\right)+(n-m) \gamma={ }_{(3)} \sum_{k=m+1}^{n}\left[u\left(\begin{array}{c}
\bar{x}_{k-1} \\
\bar{y}_{k-1}
\end{array}\right)-u\left(\begin{array}{l}
\bar{x}_{k} \\
\bar{y}_{k}
\end{array}\right)+\gamma\right] \\
& ={ }_{(4)} \sum_{k=m+1}^{n}\left[\inf _{\left(\begin{array}{c}
\bar{x} \\
\bar{y}
\end{array}\right)}\left[C\left(\left(\begin{array}{c}
\bar{x}_{k-1} \\
\bar{y}_{k-1}
\end{array}\right),\left(\begin{array}{c}
\bar{x} \\
\bar{y}
\end{array}\right)\right)+u\left(\begin{array}{c}
\bar{x} \\
\bar{y}
\end{array}\right)\right]-u\left(\begin{array}{c}
\bar{x}_{k} \\
\bar{y}_{k}
\end{array}\right)\right] \leq_{(5)} \sum_{k=m+1}^{n} C\left(\left(\begin{array}{c}
\bar{x}_{k-1} \\
\bar{y}_{k-1}
\end{array}\right),\left(\begin{array}{c}
\bar{x}_{k} \\
\bar{y}_{k}
\end{array}\right)\right) \text {, }
\end{aligned}
$$

\footnotetext{
${ }^{4}$ In the language of solid state physics, such a property means that the adapted histories behave like ground-states of one-dimensional crystal models (see, for instance, [2]).
} 
where: $={ }_{(1)}$ is due to (8); $;={ }_{(2)}$ comes from a telescopic series; $=_{(3)}$ follows again from a telescopic series, as well as from the fact that $\omega[m]=\bar{\omega}[m]$ and $\omega[n]=\bar{\omega}[n] ;=_{(4)}$ is due to (77); and $\leq_{(5)}$ follows from $\inf _{\left(\begin{array}{c}\bar{x} \\ \bar{y}\end{array}\right)}\left[C\left(\left(\begin{array}{l}\bar{x}_{k-1} \\ \bar{y}_{k-1}\end{array}\right),\left(\begin{array}{l}\bar{x} \\ \bar{y}\end{array}\right)\right)+u\left(\begin{array}{l}\bar{x} \\ \bar{y}\end{array}\right)\right] \leq C\left(\left(\begin{array}{l}\bar{x}_{k-1} \\ \bar{y}_{k-1}\end{array}\right),\left(\begin{array}{l}\bar{x}_{k} \\ \bar{y}_{k}\end{array}\right)\right)+u\left(\begin{array}{l}\bar{x}_{k} \\ \bar{y}_{k}\end{array}\right)$.

A more pertinent point about historically adapted populations is that, as we will see in the next section, they provide a theoretical argument in favor of the hypothesis of prevalence of stable sex ratios for populations under stable environmental conditions.

\section{On the existence of the asymptotic average sex ratio}

Investigating the identification of a sex ratio, we will find out that when the population maintenance cost takes into account the gender proportions, then a sex ratio will be observed in historically adapted populations. In fact, since those populations are global minimizers of cost functions, one may argue that a sex ratio will emerge as a consequence of the finiteness of available resources whenever gender densities have a linear influence on the maintenance cost.

The gender proportions of the $(i+1)^{t h}$ censused population $\left(\begin{array}{l}x_{i} \\ y_{i}\end{array}\right)$ correspond obviously to the quantities $x_{i} /\left(x_{i}+y_{i}\right)$ and $y_{i} /\left(x_{i}+y_{i}\right)$. Nevertheless, we have seen that there exists at least one historically adapted population starting from any arbitrary population. Such a fact means that all first value for a sex ratio can be then achieved and it shows that the analysis of initial data may be ineffective. Anyway, one can still ask whether a kind of sex-ratio equilibrium will be reached in the latter generations. Mathematically, it corresponds to looking for some asymptotic sex ratio, that is, given an adapted history $\omega=\left(\begin{array}{l}x_{i} \\ y_{i}\end{array}\right)_{i \in \mathbb{N}}$, to ask for the existence of the limits

$$
\lim _{i \rightarrow \infty} \frac{x_{i}}{x_{i}+y_{i}} \quad \text { and } \quad \lim _{i \rightarrow \infty} \frac{y_{i}}{x_{i}+y_{i}} .
$$

The historically-adapted-population approach does not guarantee that convergence, but it will allow to assure the convergence in average of sex ratio, namely, the existence of the limits

$$
\lim _{n \rightarrow \infty} \frac{1}{n} \sum_{k=1}^{n} \frac{x_{k}}{x_{k}+y_{k}} \quad \text { and } \quad \lim _{n \rightarrow \infty} \frac{1}{n} \sum_{k=1}^{n} \frac{y_{k}}{x_{k}+y_{k}} .
$$

More important, if the sex ratio converges in average, then there are infinitely many arbitrarily long periods of time for which it remains as close as one wants to the average limit. This mathematical property might therefore explain the documented stability of sex ratio in the nature.

\subsection{Linearly perturbed maintenance cost functions}

Let $C: \mathbb{N}^{2} \times \mathbb{N}^{2} \rightarrow \mathbb{R}$ be a cost function verifying hypotheses (11), (2) and (3). Given a vector $A=\left(a_{1}, a_{2}\right) \in \mathbb{R}^{2}$, the linearly perturbed maintenance cost function with weights $a_{1}$ and $a_{2}$ on the gender densities is the function $C_{A}: \mathbb{N}^{2} \times \mathbb{N}^{2} \rightarrow \mathbb{R}$ given by

$$
\begin{aligned}
& C_{A}\left(\left(\begin{array}{l}
x \\
y
\end{array}\right),\left(\begin{array}{l}
0 \\
0
\end{array}\right)\right)=C\left(\left(\begin{array}{l}
x \\
y
\end{array}\right),\left(\begin{array}{l}
0 \\
0
\end{array}\right)\right), \quad \text { and } \\
& C_{A}\left(\left(\begin{array}{l}
x \\
y
\end{array}\right),\left(\begin{array}{l}
\bar{x} \\
\bar{y}
\end{array}\right)\right)=C\left(\left(\begin{array}{l}
x \\
y
\end{array}\right),\left(\begin{array}{l}
\bar{x} \\
\bar{y}
\end{array}\right)\right)+a_{1} \frac{\bar{x}}{\bar{x}+\bar{y}}+a_{2} \frac{\bar{y}}{\bar{x}+\bar{y}}, \quad \text { if } \bar{x}+\bar{y}>0 .
\end{aligned}
$$


Notice that $a_{1}$ and $a_{2}$ assign cost (or benefits if negative) on the latest gender densities of the population. Besides, the original maintenance cost function $C$ could include linear and nonlinear feedbacks for the gender densities. We shall study the asymptotic average sex ratio for historically adapted populations with respect to perturbed maintenance cost functions in the above form.

It is straightforward that $C_{(0,0)}=C$. Besides, $C_{A}$ converges uniformly to $C$ as the vector $A$ tends to $(0,0)$ (see (12)). Much more crucial is the fact that hypotheses (11), (2) and (3) also hold for the perturbed cost $C_{A}$. Thus, we can apply the result of section 3.2 to get the existence of $\omega^{A}=\left(\begin{array}{l}x_{i}^{A} \\ y_{i}^{A}\end{array}\right)_{i \in \mathbb{N}} \in \Omega$, an adapted history for the maintenance cost $C_{A}$. Therefore, for a fixed cost function $C$, we can consider the map $\Gamma_{C}: \mathbb{R}^{2} \rightarrow \mathbb{R}$ given by

$$
\Gamma_{C}(A)=\lim _{n \rightarrow \infty} \frac{1}{n} \sum_{k=1}^{n} C_{A}\left(\left(\begin{array}{c}
x_{k-1}^{A} \\
y_{k-1}^{A}
\end{array}\right),\left(\begin{array}{c}
x_{k}^{A} \\
y_{k}^{A}
\end{array}\right)\right), \quad \text { for all } A \in \mathbb{R}^{2} .
$$

(The above function is well defined due to (9).)

Notice that if $\gamma$ is the minimum asymptotic average maintenance cost with respect to $C$, then clearly $\Gamma_{C}(0,0)=\gamma$. Furthermore, one can easily show that $\Gamma_{C}$ is a concave application, which, in particular, means that $\Gamma_{C}$ is continuous everywhere and differentiable almost everywhere with respect to the Lebesgue measure5. As a matter of fact, one may say a little more on the differentiable behavior of the map $\Gamma_{C}$, since one can show that

$$
\Gamma_{C}\left(a_{1}, a_{2}\right)=f_{C}\left(a_{1}-a_{2}\right)+\gamma+a_{2}, \quad \forall\left(a_{1}, a_{2}\right) \in \mathbb{R}^{2},
$$

where $f_{C}: \mathbb{R} \rightarrow \mathbb{R}$ is a concave function such that $f_{C}(0)=0$ (see Remark 6). Thus, for almost all $\Delta \in \mathbb{R}$, the map $\Gamma_{C}$ is actually differentiable along the straight line $\{(a, a-\Delta): a \in \mathbb{R}\}$.

The points of differentiability of $\Gamma_{C}$ are essential for the discussion on the existence of an asymptotic average sex ratio for historically adapted populations. Let then $\nabla \Gamma_{C}(A)$ denote the gradient vector of the function $\Gamma_{C}$ at the point $A \in \mathbb{R}^{2}$. We are able to show that, whenever $A=\left(a_{1}, a_{2}\right)$ is a point of differentiability of $\Gamma_{C}$, one necessarily has

$$
\lim _{n \rightarrow \infty} \frac{1}{n} \sum_{k=1}^{n}\left(\frac{x_{k}^{A}}{x_{k}^{A}+y_{k}^{A}}, \frac{y_{k}^{A}}{x_{k}^{A}+y_{k}^{A}}\right)=\nabla \Gamma_{C}(A)=\left(f_{C}^{\prime}\left(a_{1}-a_{2}\right), 1-f_{C}^{\prime}\left(a_{1}-a_{2}\right)\right),
$$

for any adapted history $\omega^{A}=\left(\begin{array}{c}x_{i}^{A} \\ y_{i}^{A}\end{array}\right)_{i \in \mathbb{N}} \in \Omega$ with respect to the maintenance cost function $C_{A}$. This is precisely the statement of Theorem 7 in appendix A.

First of all, (11) means that, for almost all linearly perturbed maintenance cost function, the respective historically adapted populations do share the same asymptotic average sex ratio. Moreover, this common value is constant for each family of cost functions $\left\{C_{(a, a-\Delta)}\right\}_{a \in \mathbb{R}}$, when $\Delta$ is a point of differentiability of $f_{C}$. In particular, since $f_{C}$ is concave, then $f_{C}^{\prime}$ is non-increasing whenever is defined. Therefore, if $\Delta_{1}<\Delta_{2}$ are two points of differentiability of $f_{C}$, then the asymptotic average female proportion in historically adapted populations with respect to $C_{\left(a, a-\Delta_{1}\right)}$ will not be less than the one in historically adapted populations with respect to $C_{\left(b, b-\Delta_{2}\right)}$.

\footnotetext{
${ }^{5}$ See Proposition 5 and Remark 6 in appendix A.
} 
The convergence in average of the sex ratio has a main consequence: a kind of stability along time for this biological feature. In fact, a simple lemma (see Lemma 8) assures that there will exist infinitely many arbitrarily long periods of time for which sex ratios must be as close as desired to the average limit. In more mathematical terms, suppose $A=\left(a_{1}, a_{2}\right) \in \mathbb{R}^{2}$ is a point of differentiability of the map $\Gamma_{C}$, and $\omega^{A}=\left(\begin{array}{l}x_{i}^{A} \\ y_{i}^{A}\end{array}\right)_{i \in \mathbb{N}} \in \Omega$ is an adapted history with respect to the maintenance cost function $C_{A}$. Hence, given $\epsilon>0$ arbitrarily small and $M>0$ as large as one wants, there are infinitely many finite histories $\omega^{A}[m, n]=\left(\begin{array}{l}x_{i}^{A} \\ y_{i}^{A}\end{array}\right)_{m \leq i \leq n}$ such that $n-m \geq M$ and

$$
\left|\sum_{k=m+1}^{n}\left(\frac{x_{k}^{A}}{x_{k}^{A}+y_{k}^{A}}-f_{C}^{\prime}\left(a_{1}-a_{2}\right)\right)\right|<\epsilon
$$

This property might clearly provide a reasonable explanation for the observed stability of sex ratio in nature, underlying the major role of the non-trivial interaction between a finite-resource environment and the growth and maintenance of a two-sex population.

\subsection{From perturbed to non-perturbed maintenance costs}

We have an asymptotic average sex ratio for historically adapted populations with respect to almost all linearly perturbed maintenance cost function. However, $\Gamma_{C}$ may be non-differentiable along countably many straight lines $\{(b, b-\Delta): b \in \mathbb{R}\}$, and the limit (11) is only guaranteed if $A \in \mathbb{R}^{2}$ is a point of differentiability of $\Gamma_{C}$. Note that this limit could exist for some point of nondifferentiability of $\Gamma_{C}$, but the argumentation in the proof of Theorem 7 cannot be used to decide if this is the case. In particular, one cannot assure that $\Gamma_{C}$ is differentiable at $A=(0,0)$, which would imply the existence of an asymptotic average sex ratio for historically adapted populations with respect to the original non-perturbed maintenance cost function $C$.

Although we cannot always guarantee the existence of an asymptotic average sex ratio, the characterization of $\Gamma_{C}$ given in (10) allows us to deduce that even in the worst case the average sex ratio takes values in some fixed interval (see Theorem 10). As a matter of fact, for all $B=$ $(b, b-\Delta) \in \mathbb{R}^{2}$, there exist real constants $L_{\Delta}$ and $R_{\Delta}$ (depending only on the cost function $C$ and on the real parameter $\Delta$ ) such that

$$
0 \leq L_{\Delta} \leq \liminf _{n \rightarrow \infty} \frac{1}{n} \sum_{k=1}^{n} \frac{x_{k}^{B}}{x_{k}^{B}+y_{k}^{B}} \leq \limsup _{n \rightarrow \infty} \frac{1}{n} \sum_{k=1}^{n} \frac{x_{k}^{B}}{x_{k}^{B}+y_{k}^{B}} \leq R_{\Delta} \leq 1,
$$

whenever $\omega^{B}=\left(\begin{array}{c}x_{i}^{B} \\ y_{i}^{B}\end{array}\right)_{i \in \mathbb{N}} \in \Omega$ is an adapted history for the maintenance cost function $C_{B}$.

For any points $B=(b, b-\Delta)$ and $\bar{B}=(\beta, \beta-\bar{\Delta})$ with $\Delta<\bar{\Delta}$, one can show that $R_{\bar{\Delta}} \leq L_{\Delta}$ (see Remark 11). Hence the respective intervals $\left[L_{\Delta}, R_{\Delta}\right]$ and $\left[L_{\bar{\Delta}}, R_{\bar{\Delta}}\right]$ may intersect each other only at their common boundary. In particular, a small perturbation, let us say, on $\bar{\Delta}$ will imply that the corresponding average sex ratios of historically adapted populations must take its values outside the interval $\left(L_{\bar{\Delta}}, R_{\bar{\Delta}}\right)$. One might interpret this fact as a kind of instability of average sex ratios for points of non-differentiability of $\Gamma_{C}$. 
To illustrate the above discussion, let us consider an extreme situation. Suppose that there exists some point $\bar{B}=(\beta, \beta-\bar{\Delta})$ such that $L_{\bar{\Delta}}=0$ and $R_{\bar{\Delta}}=1$. It is straightforward that, for any other point $B=(b, b-\Delta)$ with $\Delta \neq \bar{\Delta}$, we have

$$
\text { either } \quad \lim _{n \rightarrow \infty} \frac{1}{n} \sum_{k=1}^{n} \frac{x_{k}^{B}}{x_{k}^{B}+y_{k}^{B}}=0 \quad \text { or } \quad \lim _{n \rightarrow \infty} \frac{1}{n} \sum_{k=1}^{n} \frac{x_{k}^{B}}{x_{k}^{B}+y_{k}^{B}}=1 \text {, }
$$

for all adapted history $\omega^{B}=\left(\begin{array}{c}x_{i}^{B} \\ y_{i}^{B}\end{array}\right)_{i \in \mathbb{N}} \in \Omega$ with respect to $C_{B}$. So only for cost functions $C_{(\bar{b}, \bar{b}-\bar{\Delta})}$ it could exist historically adapted populations with two genders coexisting as time goes by.

A brief concluding remark is that, for the special case of the non-perturbed maintenance cost function $C=C_{(0,0)}$, there always exist constants $0 \leq L_{0} \leq R_{0} \leq 1$ such that average sex ratios of historically adapted populations for $C$ either converge to some point of the interval $\left[L_{0}, R_{0}\right]$ or take values in this interval in a periodic or random way, without convergence.

\section{Discussion}

We proposed a new theoretical paradigm for sex-ratio problems: reproductive interactions are supposed to have interconnectedness governed by a maintenance cost function depending explicitly on the size of male and female populations. By considering an environment with finite resources, we are compelled to take three hypotheses on the maintenance cost function: there exists a minimum cost (or a maximum benefit) which could be achieved by some population; the cost diverges to infinity as the latest population increases; the cost is dominated by the current population size. In this framework, we were able to show that there always exist historically adapted populations, which are populations minimizing the maintenance cost along time. Furthermore, the main result established here has guaranteed that, for almost all linearly perturbed maintenance cost function, the average sex ratios of the respective historically adapted populations do converge.

We emphasize that the proposed model has mainly a heuristic focus and does not try to explain mechanisms which could lead some population to be historically adapted. One obviously recognizes the fundamental importance of biological researches into a possible validation of such an approach. The main point seems to be an affirmative answer to the challenge of representing interactions of an actual biological population through a maintenance cost function with the desired features.

We notice that formalism developed in previous sections and its consequences can be immediately generalized to various other situations. We would like to briefly discuss some of them.

Cost dependence on a finite number of consecutive censuses. One may consider a maintenance cost function $C: \mathbb{N}^{2 L} \rightarrow \mathbb{R}$ depending on $L \geq 2$ consecutive population census, which can be seen again depending on two coordinates $C: \mathbb{N}^{2(L-1)} \times \mathbb{N}^{2(L-1)} \rightarrow \mathbb{R}$ via the identification

$$
C\left(\left(\begin{array}{l}
x_{1} \\
y_{1}
\end{array}\right),\left(\begin{array}{l}
x_{2} \\
y_{2}
\end{array}\right), \ldots,\left(\begin{array}{l}
x_{L} \\
y_{L}
\end{array}\right)\right)=C\left(\left(\begin{array}{l}
\left(\begin{array}{l}
x_{1} \\
y_{1}
\end{array}\right)\left(\begin{array}{l}
x_{2} \\
y_{2}
\end{array}\right) \\
\ldots
\end{array}\right.\right.
$$

One may now use such a point of view to rewrite the assumptions on the cost function and to easily obtain the analogous results for historically adapted populations. 
Age-structured population models. We can introduce, for instance, the quantities of newborns of each gender. Hence, if newborns are included as a cost factor, then, for almost all perturbed cost function, there shall exist an identifiable primary sex ratio in historically adapted populations. More generally, one may analyze a maintenance cost function depending on $M \geq 2$ age classes for both genders, namely, a function $C: \mathbb{N}^{2 M} \times \mathbb{N}^{2 M} \rightarrow \mathbb{R}, C\left(\left(\begin{array}{c}\left(x_{1}, x_{2}, \ldots, x_{M}\right) \\ \left(y_{1}, y_{2}, \ldots, y_{M}\right)\end{array}\right),\left(\begin{array}{c}\left.\left(\begin{array}{c}\left.\bar{x}_{1}, \bar{x}_{2}, \ldots, \bar{x}_{M}\right) \\ \left(\bar{y}_{1}, \bar{y}_{2}, \ldots, \bar{y}_{M}\right)\end{array}\right)\right) \\ y_{1}\end{array}\right)\right.$.

Sequential and simultaneous hermaphroditism. By adding variables in our model, we can without difficulty extend our study to the occurrence at the same time of separate and combined sexes in some biological system. For instance, if $X$ and $Y$ denote the sizes of the dioecious part of the population, concerning the sex reversal part, let $h_{x}$ and $H_{y}$ be the number of sequential hermaphrodites reproducing early in life, respectively, as females and as males. The number of individuals after sex changes will be indicated then by $h^{y}$ and $H^{x}$, respectively. At last, let $Z$ denote the number of individuals having simultaneously both male and female reproductive organs. Therefore, the distribution of dioecy versus hermaphroditism can be investigated, for example, by the means of a maintenance cost function $C: \mathbb{N}^{7} \times \mathbb{N}^{7} \rightarrow \mathbb{R}$,

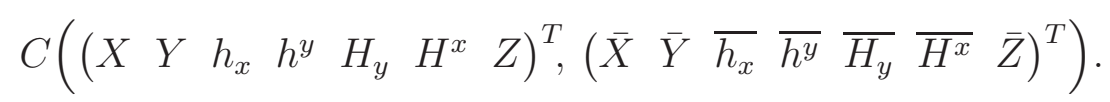

Periodic cost functions. The population maintenance cost may vary periodically along time. Such a situation corresponds to consider a family of cost functions $C_{1}, C_{2}, \ldots, C_{N}: \mathbb{N}^{2} \times \mathbb{N}^{2} \rightarrow \mathbb{R}$ and, for any finite history $\omega[m, n]=\left(\begin{array}{l}x_{i} \\ y_{i}\end{array}\right)_{m<i<n}$, a total cost $\sum_{k=m+1}^{n} C_{k-1(\bmod N)}\left(\left(\begin{array}{l}x_{k-1} \\ y_{k-1}\end{array}\right),\left(\begin{array}{l}x_{k} \\ y_{k}\end{array}\right)\right)$. The analysis of the periodic case may be reduced to our time-independent case just by introducing a conjunction cost map

$C\left(\left(\begin{array}{l}x \\ y\end{array}\right),\left(\begin{array}{l}\bar{x} \\ \bar{y}\end{array}\right)\right):=\inf \left(\begin{array}{l}x_{1} \\ y_{1}\end{array}\right),\left(\begin{array}{l}x_{2} \\ y_{2}\end{array}\right), \ldots,\left(\begin{array}{l}x_{N-1} \\ x_{N-1}\end{array}\right) \in \mathbb{N}^{2}\left[C_{1}\left(\left(\begin{array}{l}x \\ y\end{array}\right),\left(\begin{array}{l}x_{1} \\ y_{1}\end{array}\right)\right)+C_{2}\left(\left(\begin{array}{l}x_{1} \\ y_{1}\end{array}\right),\left(\begin{array}{l}x_{2} \\ y_{2}\end{array}\right)\right)+\ldots+C_{N}\left(\left(\begin{array}{l}x_{N-1} \\ y_{N-1}\end{array}\right),\left(\begin{array}{l}\bar{x} \\ \bar{y}\end{array}\right)\right)\right]$.

It would certainly be very interesting to take into account two or more generalizations at the same application. For instance, an age-structured model with sex reversal individuals might help to understand whether there should exist a special age for sex change. Here again, from the biological perspective, in this particular situation as well as in many other examples of potential applications, such a form of modeling requires first to describe more explicitly properties of a maintenance cost function regulating the reproductive interactions of a given population.

\section{Appendix A}

In this appendix we shall give the mathematical proofs of the results previously discussed. From now on, let $C: \mathbb{N}^{2} \times \mathbb{N}^{2} \rightarrow \mathbb{R}$ be a function verifying assumptions (11), (2) and (3). The main idea is to associate to such a maintenance cost function a kind of Lax-Oleinik operator and use its fixed points to construct historically adapted populations as well as to study their asymptotic properties. Lax-Oleinik fixed point techniques have been successfully explored in several areas. A very important example comes from calculus of variations: the Lax-Oleinik semigroup, which is essential in Fathi's weak KAM theory for Lagrangian mechanics (see [6]). 
First of all, we need to introduce the spaces on which our Lax-Oleinik operator will act. Denote by $\ell^{\infty}\left(\mathbb{N}^{2}\right)$ the set of all real valued bounded functions on $\mathbb{N}^{2}$, and denote by $\ell^{\infty}\left(\mathbb{N}^{2}\right) / \mathbb{R}$ the set of all real valued bounded functions on $\mathbb{N}^{2}$ modulo constants, that is, the set of equivalence classes $[f]:=\left\{g \in \ell^{\infty}\left(\mathbb{N}^{2}\right): f-g \equiv c t e\right\}$. Both $\ell^{\infty}\left(\mathbb{N}^{2}\right)$ and $\ell^{\infty}\left(\mathbb{N}^{2}\right) / \mathbb{R}$ are Banach spaces with norms $\|f\|_{\infty}:=\sup _{\left(\begin{array}{l}x \\ y\end{array}\right) \in \mathbb{N}^{2}}\left|f\left(\begin{array}{l}x \\ y\end{array}\right)\right|$ and $\|[f]\|_{\#}:=\inf _{\kappa \in \mathbb{R}}\|f+\kappa\|_{\infty}$, respectively.

Definition 2 (The Lax-Oleinik operator). Let $T_{C}$ be the operator acting on $\ell^{\infty}\left(\mathbb{N}^{2}\right)$ by

$$
T_{C} f\left(\begin{array}{l}
x \\
y
\end{array}\right):=\inf _{\left(\begin{array}{c}
\bar{x} \\
\bar{y}
\end{array}\right) \in \mathbb{N}^{2}}\left[C\left(\left(\begin{array}{l}
x \\
y
\end{array}\right),\left(\begin{array}{l}
\bar{x} \\
\bar{y}
\end{array}\right)\right)+f\left(\begin{array}{l}
\bar{x} \\
\bar{y}
\end{array}\right)\right], \quad \forall\left(\begin{array}{l}
x \\
y
\end{array}\right) \in \mathbb{N}^{2},
$$

whenever $f$ is a real valued bounded function on $\mathbb{N}^{2}$.

Notice that the operator $T_{C}$ is well defined, since

$$
\inf C-\|f\|_{\infty} \leq T_{C} f \leq \sup _{\left(\begin{array}{l}
x \\
y
\end{array}\right) \in \mathbb{N}^{2}} C\left(\left(\begin{array}{l}
x \\
y
\end{array}\right),\left(\begin{array}{l}
0 \\
0
\end{array}\right)\right)+\|f\|_{\infty} \leq \mathfrak{K}_{c}+C\left(\left(\begin{array}{l}
0 \\
0
\end{array}\right),\left(\begin{array}{l}
0 \\
0
\end{array}\right)\right)+\|f\|_{\infty} .
$$

The above upper bound also implies that the infimum in the definition of the Lax-Oleinik operator is actually a minimum. Indeed, as the cost function $C$ verifies hypothesis (2),$T_{C} f\left(\begin{array}{l}x \\ y\end{array}\right)$ will be selected among a finite number of values. Furthermore, note that $T_{C}(f+\kappa)=T_{C}(f)+\kappa$ for any $\kappa \in \mathbb{R}$. Thus, we can consider $T_{C}$ acting on $\ell^{\infty}\left(\mathbb{N}^{2}\right) / \mathbb{R}$.

Theorem 3. The operator $T_{C}: \ell^{\infty}\left(\mathbb{N}^{2}\right) / \mathbb{R} \rightarrow \ell^{\infty}\left(\mathbb{N}^{2}\right) / \mathbb{R}$ has a fixed point.

Proof. We remark first that

$$
2\|[f]\|_{\#}=\operatorname{osc}(f):=\sup _{\left(\begin{array}{c}
x \\
y
\end{array}\right),\left(\begin{array}{l}
\bar{y} \\
\bar{y}
\end{array}\right) \in \mathbb{N}^{2}}\left[f\left(\begin{array}{l}
x \\
y
\end{array}\right)-f\left(\begin{array}{l}
\bar{x} \\
\bar{y}
\end{array}\right)\right]
$$

where $f$ is any element of the equivalence class $[f]$.

Given $\lambda \in(0,1)$, let $M_{\lambda}$ be the multiplication by $1-\lambda$ acting on $\ell^{\infty}\left(\mathbb{N}^{2}\right) / \mathbb{R}$. The operator $T_{C} \circ M_{\lambda}$ is a contraction and has therefore a fixed point $\left[u_{\lambda}\right] \in \ell^{\infty}\left(\mathbb{N}^{2}\right) / \mathbb{R}$, that is, $\left(T_{C} \circ M_{\lambda}\right)\left[u_{\lambda}\right]=$ $T_{C}\left[(1-\lambda) u_{\lambda}\right]=\left[u_{\lambda}\right]$. Hence, observe that

$$
\left\|\left[u_{\lambda}\right]\right\|_{\#}=\frac{1}{2} \operatorname{osc}\left(\left(T_{C} \circ M_{\lambda}\right) u_{\lambda}\right)=\frac{1}{2} \operatorname{osc}\left(T_{C}(1-\lambda) u_{\lambda}\right) \leq \frac{\mathfrak{K}_{C}}{2} .
$$

In particular, the family $\left\{\left[u_{\lambda}\right]\right\}_{\lambda \in(0,1)}$ has an accumulation point $[u] \in \ell^{\infty}\left(\mathbb{N}^{2}\right) / \mathbb{R}$ as $\lambda$ goes to zero. Choose $\lambda_{i} \rightarrow 0$ such that $\left[u_{\lambda_{i}}\right] \rightarrow[u]$ as $i \rightarrow \infty$. Since $T_{C}$ is 1-Lipschitz, we have

$$
T_{C}[u]=\lim _{i \rightarrow+\infty} T_{C}\left[\left(1-\lambda_{i}\right) u_{\lambda_{i}}\right]=\lim _{i \rightarrow+\infty}\left[u_{\lambda_{i}}\right]=[u] .
$$


Remark 4. We have $T_{C}[u]=[u]$ for some equivalence class $[u] \in \ell^{\infty}\left(\mathbb{N}^{2}\right) / \mathbb{R}$. Therefore, if $u \in \ell^{\infty}\left(\mathbb{N}^{2}\right)$ is an element of the equivalence class $[u]$, it follows that

$$
u\left(\begin{array}{l}
x \\
y
\end{array}\right)+\gamma=T_{C} u\left(\begin{array}{l}
x \\
y
\end{array}\right)=\min _{\left(\begin{array}{c}
\bar{x} \\
\bar{y}
\end{array}\right) \in \mathbb{N}^{2}}\left[C\left(\left(\begin{array}{l}
x \\
y
\end{array}\right),\left(\begin{array}{l}
\bar{x} \\
\bar{y}
\end{array}\right)\right)+u\left(\begin{array}{l}
\bar{x} \\
\bar{y}
\end{array}\right)\right], \quad \forall\left(\begin{array}{l}
x \\
y
\end{array}\right) \in \mathbb{N}^{2},
$$

for some real constant $\gamma$. Recall that such a functional equation allows to construct an adapted history $\omega=\left(\begin{array}{l}x_{i} \\ y_{i}\end{array}\right)_{i \in \mathbb{N}}$ starting with any given initial population $\left(\begin{array}{l}x_{0} \\ y_{0}\end{array}\right)$. In particular, it is easy to see that, for any adapted history $\omega=\left(\begin{array}{l}x_{i} \\ y_{i}\end{array}\right)_{i \in \mathbb{N}}$ and all arbitrary history $\bar{\omega}=\left(\begin{array}{c}\bar{x}_{i} \\ \bar{y}_{i}\end{array}\right)_{i \in \mathbb{N}}$,

$$
\begin{aligned}
\lim _{n \rightarrow \infty} \frac{1}{n} \sum_{k=1}^{n}\left[C\left(\left(\begin{array}{l}
x_{k-1} \\
y_{k-1}
\end{array}\right),\left(\begin{array}{l}
x_{k} \\
y_{k}
\end{array}\right)\right)+u\left(\begin{array}{l}
x_{k} \\
y_{k}
\end{array}\right)-u\left(\begin{array}{l}
x_{k-1} \\
y_{k-1}
\end{array}\right)\right]= \\
=\gamma \leq \liminf _{n \rightarrow \infty} \frac{1}{n} \sum_{k=1}^{n}\left[C\left(\left(\begin{array}{l}
\bar{x}_{k-1} \\
\bar{y}_{k-1}
\end{array}\right),\left(\begin{array}{l}
\bar{x}_{k} \\
\bar{y}_{k}
\end{array}\right)\right)+u\left(\begin{array}{l}
\bar{x}_{k} \\
\bar{y}_{k}
\end{array}\right)-u\left(\begin{array}{l}
\bar{x}_{k-1} \\
\bar{y}_{k-1}
\end{array}\right)\right] .
\end{aligned}
$$

Thus, one clearly has (9) and

$$
\gamma=\inf _{\left(\begin{array}{c}
\bar{x}_{i} \\
\bar{y}_{i}
\end{array}\right)_{i \in \mathbb{N}} \in \Omega} \liminf _{n \rightarrow \infty} \frac{1}{n} \sum_{k=1}^{n} C\left(\left(\begin{array}{c}
\bar{x}_{k-1} \\
\bar{y}_{k-1}
\end{array}\right),\left(\begin{array}{c}
\bar{x}_{k} \\
\bar{y}_{k}
\end{array}\right)\right) .
$$

Now, given $A \in \mathbb{R}^{2}$, consider the perturbed maintenance cost

$$
C_{A}\left(\left(\begin{array}{l}
x \\
y
\end{array}\right),\left(\begin{array}{l}
\bar{x} \\
\bar{y}
\end{array}\right)\right):=C\left(\left(\begin{array}{l}
x \\
y
\end{array}\right),\left(\begin{array}{l}
\bar{x} \\
\bar{y}
\end{array}\right)\right)+\left\langle A,\left(\frac{\bar{x}}{\bar{x}+\bar{y}}, \frac{\bar{y}}{\bar{x}+\bar{y}}\right)\right\rangle,
$$

with the convention that zero over zero is equal to zero. It is straightforward that $C_{A}$ verifies (11), (2) and (3), and $\mathfrak{K}_{C_{A}}=\mathfrak{K}_{C}$. Furthermore

$$
\left\|C_{A}-C_{B}\right\|_{\infty}=\sup _{\left(\begin{array}{l}
\bar{x} \\
\bar{y}
\end{array}\right) \in \mathbb{N}^{2}}\left|\left\langle A-B,\left(\frac{\bar{x}}{\bar{x}+\bar{y}}, \frac{\bar{y}}{\bar{x}+\bar{y}}\right)\right\rangle\right| \leq\|A-B\|, \quad \forall A, B \in \mathbb{R}^{2} .
$$

Let $\Gamma_{C}: \mathbb{R}^{2} \rightarrow \mathbb{R}$ be the map defined by

$$
\Gamma_{C}(A):=\inf _{\left(\begin{array}{c}
\bar{x}_{i} \\
\left.\bar{y}_{i}\right)_{i \in \mathbb{N}} \in \Omega
\end{array}\right.} \liminf _{n \rightarrow \infty} \frac{1}{n} \sum_{k=1}^{n} C_{A}\left(\left(\begin{array}{c}
\bar{x}_{k-1} \\
\bar{y}_{k-1}
\end{array}\right),\left(\begin{array}{c}
\bar{x}_{k} \\
\bar{y}_{k}
\end{array}\right)\right) .
$$

Obviously $\Gamma_{C}(0,0)=\gamma$. Moreover, we have that

Proposition 5. The function $\Gamma_{C}$ is concave.

Proof. Given $A, B \in \mathbb{R}^{2}$ and $t \in[0,1]$, let $\omega=\left(\begin{array}{l}x_{i} \\ y_{i}\end{array}\right)_{i \in \mathbb{N}}$ be an adapted history with respect to the cost function $C_{t A+(1-t) B}$. Therefore, by the very definition of $\Gamma_{C}$, we get

$$
\begin{aligned}
t \Gamma_{C}(A)+(1-t) \Gamma_{C}(B) & \leq \lim _{n \rightarrow \infty} \frac{1}{n} \sum_{k=1}^{n}\left[t C_{A}\left(\left(\begin{array}{l}
x_{k-1} \\
y_{k-1}
\end{array}\right),\left(\begin{array}{l}
x_{k} \\
y_{k}
\end{array}\right)\right)+(1-t) C_{B}\left(\left(\begin{array}{l}
x_{k-1} \\
y_{k-1}
\end{array}\right),\left(\begin{array}{l}
x_{k} \\
y_{k}
\end{array}\right)\right)\right] \\
& =\lim _{n \rightarrow \infty} \frac{1}{n} \sum_{k=1}^{n} C_{t A+(1-t) B}\left(\left(\begin{array}{l}
x_{k-1} \\
y_{k-1}
\end{array}\right),\left(\begin{array}{l}
x_{k} \\
y_{k}
\end{array}\right)\right)=\Gamma_{C}(t A+(1-t) B) .
\end{aligned}
$$


Remark 6. A real valued concave function on $\mathbb{R}^{n}$ is locally Lipschitz continuou 6 and hence, by Rademacher's theorem, differentiable almost everywhere with respect to the Lebesgue measure. Thus, Theorem 5 implies that Lebesgue-almost every $A \in \mathbb{R}^{2}$ is a point of differentiability of the map $\Gamma_{C}$. As a matter of fact, one may be a little more precise on the description of the points of differentiability of $\Gamma_{C}$. To that end, notice that we can write

$$
\Gamma_{C}\left(a_{1}, a_{2}\right)=f_{C}\left(a_{1}-a_{2}\right)+\gamma+a_{2}, \quad \forall\left(a_{1}, a_{2}\right) \in \mathbb{R}^{2},
$$

with $f_{C}: \mathbb{R} \rightarrow \mathbb{R}$ defined by

$$
f_{C}(\Delta):=\inf _{\left(\begin{array}{c}
\bar{x}_{i} \\
\bar{y}_{i}
\end{array}\right)_{i \in \mathbb{N}} \in \Omega} \liminf _{n \rightarrow \infty} \frac{1}{n} \sum_{k=1}^{n}\left[C\left(\left(\begin{array}{c}
\bar{x}_{k-1} \\
\bar{y}_{k-1}
\end{array}\right),\left(\begin{array}{c}
\bar{x}_{k} \\
\bar{y}_{k}
\end{array}\right)\right)+\frac{\bar{x}_{k}}{\bar{x}_{k}+\bar{y}_{k}} \Delta-\gamma\right], \quad \forall \Delta \in \mathbb{R} .
$$

Certainly $f_{C}(0)=0$. Moreover, as in the proof of Proposition 5, one may immediately verify that the function $f_{C}$ is concave and therefore differentiable almost everywhere with respect to the Lebesgue measure on the real line. So we conclude that, for Lebesgue-almost every $\Delta \in \mathbb{R}$, the map $\Gamma_{C}$ is indeed differentiable along the straight line $\{(a, a-\Delta): a \in \mathbb{R}\}$.

The next theorem shows that points of differentiability of $\Gamma_{C}$ play a crucial role on the study of average sex ratio for historically adapted populations. Its proof is very similar to Gomes' argument for the asymptotic behavior of optimal trajectories defined by discrete viscosity solutions (see [8]).

Theorem 7. Let $A=\left(a_{1}, a_{2}\right) \in \mathbb{R}^{2}$ be a point of differentiability of $\Gamma_{C}$, and let $\omega^{A}=\left(\begin{array}{l}x_{i}^{A} \\ y_{i}^{A}\end{array}\right)_{i \in \mathbb{N}}$ be an adapted history for the maintenance cost $C_{A}$. Then, one has

$$
\lim _{n \rightarrow \infty} \frac{1}{n} \sum_{k=1}^{n}\left(\frac{x_{k}^{A}}{x_{k}^{A}+y_{k}^{A}}, \frac{y_{k}^{A}}{x_{k}^{A}+y_{k}^{A}}\right)=\nabla \Gamma_{C}(A)=\left(f_{C}^{\prime}\left(a_{1}-a_{2}\right), 1-f_{C}^{\prime}\left(a_{1}-a_{2}\right)\right) .
$$

Proof. Let $u_{A} \in \ell^{\infty}\left(\mathbb{N}^{2}\right)$ be such that $u_{A}\left(\begin{array}{l}x_{i}^{A} \\ y_{i}^{A}\end{array}\right)+\Gamma_{C}(A)=T_{C_{A}} u_{A}\left(\begin{array}{l}x_{i}^{A} \\ y_{i}^{A}\end{array}\right)=C_{A}\left(\left(\begin{array}{l}x_{i}^{A} \\ y_{i}^{A}\end{array}\right),\left(\begin{array}{l}x_{i+1}^{A} \\ y_{i+1}^{A}\end{array}\right)\right)+u_{A}\left(\begin{array}{l}x_{i+1}^{A} \\ y_{i+1}^{A}\end{array}\right)$, for all $i \in \mathbb{N}$. Therefore, for all $n \geq 1$, we obtain

$$
u_{A}\left(\begin{array}{c}
x_{0}^{A} \\
y_{0}^{A}
\end{array}\right)=\sum_{k=1}^{n} C_{A}\left(\left(\begin{array}{c}
x_{k-1}^{A} \\
y_{k-1}^{A}
\end{array}\right),\left(\begin{array}{c}
x_{k}^{A} \\
y_{k}^{A}
\end{array}\right)\right)+u_{A}\left(\begin{array}{c}
x_{n}^{A} \\
y_{n}^{A}
\end{array}\right)-n \Gamma_{C}(A) .
$$

For $h>0$ and $B \in \mathbb{R}^{2}$, let $u_{A+h B} \in \ell^{\infty}\left(\mathbb{N}^{2}\right)$ be such that $T_{C_{A+h B}} u_{A+h B}=u_{A+h B}+\Gamma_{C}(A+h B)$. It is straightforward that

$$
u_{A+h B}\left(\begin{array}{c}
x_{0}^{A} \\
y_{0}^{A}
\end{array}\right) \leq \sum_{k=1}^{n} C_{A+h B}\left(\left(\begin{array}{c}
x_{k-1}^{A} \\
y_{k-1}^{A}
\end{array}\right),\left(\begin{array}{c}
x_{k}^{A} \\
y_{k}^{A}
\end{array}\right)\right)+u_{A+h B}\left(\begin{array}{c}
x_{n}^{A} \\
y_{n}^{A}
\end{array}\right)-n \Gamma_{C}(A+h B), \quad \forall n \geq 1 .
$$

Thus, clearly

$$
\begin{aligned}
& u_{A+h B}\left(\begin{array}{c}
x_{0}^{A} \\
y_{0}^{A}
\end{array}\right)-u_{A}\left(\begin{array}{c}
x_{0}^{A} \\
y_{0}^{A}
\end{array}\right) \leq \\
& \quad \leq h \sum_{k=1}^{n}\left\langle B,\left(\frac{x_{k}^{A}}{x_{k}^{A}+y_{k}^{A}}, \frac{y_{k}^{A}}{x_{k}^{A}+y_{k}^{A}}\right)\right\rangle-n\left(\Gamma_{C}(A+h B)-\Gamma_{C}(A)\right)+u_{A+h B}\left(\begin{array}{l}
x_{n}^{A} \\
y_{n}^{A}
\end{array}\right)-u_{A}\left(\begin{array}{c}
x_{n}^{A} \\
y_{n}^{A}
\end{array}\right) .
\end{aligned}
$$

${ }^{6}$ Actually, it is not hard to directly check that, for all $A, B \in \mathbb{R}^{2}$, we have $\left|\Gamma_{C}(A)-\Gamma_{C}(B)\right| \leq\|A-B\|$. 
Since $u_{A+h B}\left(\begin{array}{c}x_{0}^{A} \\ y_{0}^{A}\end{array}\right)-u_{A+h B}\left(\begin{array}{c}x_{n}^{A} \\ y_{n}^{A}\end{array}\right)+u_{A}\left(\begin{array}{c}x_{n}^{A} \\ y_{n}^{A}\end{array}\right)-u_{A}\left(\begin{array}{c}x_{0}^{A} \\ y_{0}^{A}\end{array}\right) \geq-\operatorname{osc}\left(T_{C_{A+h B}} u_{A+h B}\right)-\operatorname{osc}\left(T_{C_{A}} u_{A}\right) \geq-2 \mathfrak{K}_{C}$, it follows that

$$
-\frac{2 \mathfrak{K}_{C}}{h n}+\frac{\Gamma_{C}(A+h B)-\Gamma_{C}(A)}{h} \leq\left\langle B, \frac{1}{n} \sum_{k=1}^{n}\left(\frac{x_{k}^{A}}{x_{k}^{A}+y_{k}^{A}}, \frac{y_{k}^{A}}{x_{k}^{A}+y_{k}^{A}}\right)\right\rangle .
$$

The same argument can be applied to $-B$ and hence we also deduce that

$$
\left\langle B, \frac{1}{n} \sum_{k=1}^{n}\left(\frac{x_{k}^{A}}{x_{k}^{A}+y_{k}^{A}}, \frac{y_{k}^{A}}{x_{k}^{A}+y_{k}^{A}}\right)\right\rangle \leq \frac{2 \mathfrak{K}_{C}}{h n}-\frac{\Gamma_{C}(A-h B)-\Gamma_{C}(A)}{h} .
$$

So setting $h=m / n$ for a fixed $m>0$ and taking $n \rightarrow \infty$, as $A$ is a point of differentiability of $\Gamma_{C}$, from the last two inequalities we get that

$$
\begin{aligned}
-\frac{2 \mathfrak{K}_{C}}{m}+\left\langle B, \nabla \Gamma_{C}(A)\right\rangle \leq & \left\langle B, \liminf _{n \rightarrow \infty} \frac{1}{n} \sum_{k=1}^{n}\left(\frac{x_{k}^{A}}{x_{k}^{A}+y_{k}^{A}}, \frac{y_{k}^{A}}{x_{k}^{A}+y_{k}^{A}}\right)\right\rangle \leq \\
& \leq\left\langle B, \limsup _{n \rightarrow \infty} \frac{1}{n} \sum_{k=1}^{n}\left(\frac{x_{k}^{A}}{x_{k}^{A}+y_{k}^{A}}, \frac{y_{k}^{A}}{x_{k}^{A}+y_{k}^{A}}\right)\right\rangle \leq \frac{2 \mathfrak{K}_{C}}{m}+\left\langle B, \nabla \Gamma_{C}(A)\right\rangle .
\end{aligned}
$$

Finally, taking $m \rightarrow+\infty$, we obtain

$$
\left\langle B, \lim _{n \rightarrow \infty} \frac{1}{n} \sum_{k=1}^{n}\left(\frac{x_{k}^{A}}{x_{k}^{A}+y_{k}^{A}}, \frac{y_{k}^{A}}{x_{k}^{A}+y_{k}^{A}}\right)\right\rangle=\left\langle B, \nabla \Gamma_{C}(A)\right\rangle,
$$

and then, since the equality holds for all $B \in \mathbb{R}^{2}$, we conclude that

$$
\lim _{n \rightarrow \infty} \frac{1}{n} \sum_{k=1}^{n}\left(\frac{x_{k}^{A}}{x_{k}^{A}+y_{k}^{A}}, \frac{y_{k}^{A}}{x_{k}^{A}+y_{k}^{A}}\right)=\nabla \Gamma_{C}(A) .
$$

Despite of being a weak form of convergence, in fact convergence in average underlines a recurrence property of the sequence. More precisely, we have the following result from real analysis.

Lemma 8. Let $\left\{\alpha_{i}\right\} \subset \mathbb{R}$ be a sequence such that $\lim _{n \rightarrow \infty}(1 / n) \sum_{k=1}^{n} \alpha_{k}=\alpha \in \mathbb{R}$. Let $\mathbb{I} \subset \mathbb{N}$ be $a$ subset of positive density, that is,

$$
\lim _{n \rightarrow \infty} \frac{\#\{k \in \mathbb{I}: 1 \leq k \leq n\}}{n}=: \beta>0 .
$$

Then, for all $\epsilon>0$ and for any integer $L>0$, there exist $m, n \in \mathbb{I}$, with $n>m \geq L$, such that

$$
\left|\sum_{k=m+1}^{n}\left(\alpha_{k}-\alpha\right)\right|<\epsilon
$$


For the convenience of the reader, we give a short proof of this lemma.

Proof. Without loss of generality, we can assume that $\alpha=0$. Fix $\rho \in(0, \epsilon \beta / 8)$. There exists then a positive integer $n_{0} \in \mathbb{I}$ such that

$$
\#\{k \in \mathbb{I}: 1 \leq k \leq n\} \geq \frac{\beta n}{2} \quad \text { and } \quad\left|\sum_{k=1}^{n} \alpha_{k}\right| \leq \rho n, \quad \forall n \geq n_{0} .
$$

We may suppose that $n_{0} \geq L$. Clearly $\left\{\sum_{k=1}^{n} \alpha_{k}: n_{0} \leq n \leq n_{1}\right\} \subset\left[-\rho n_{1}, \rho n_{1}\right]$. Considering thus

$$
n_{1} \in \mathbb{N} \quad \text { with } \quad n_{1}>\max \left\{n_{0}, \frac{4}{\beta} \#\left\{k \in \mathbb{I}: 1 \leq k \leq n_{0}\right\}\right\}
$$

we assure that

$$
\#\left\{k \in \mathbb{I}: n_{0}<k \leq n_{1}\right\}=\#\left\{k \in \mathbb{I}: 1 \leq k \leq n_{1}\right\}-\#\left\{k \in \mathbb{I}: 1 \leq k \leq n_{0}\right\}>\frac{\beta n_{1}}{2}-\frac{\beta n_{1}}{4}=\frac{\beta n_{1}}{4} .
$$

By the pigeonhole principle, there must be $m, n \in \mathbb{I} \cap\left\{n_{0}, n_{0}+1, \ldots, n_{1}\right\}$, with $n>m$, such that

$$
\begin{aligned}
\left|\sum_{k=m+1}^{n} \alpha_{k}\right|=\left|\sum_{k=1}^{m} \alpha_{k}-\sum_{k=1}^{n} \alpha_{k}\right| \leq \frac{2 \rho n_{1}}{\#\left\{k \in \mathbb{I}: n_{0} \leq k \leq n_{1}\right\}-1}= \\
=\frac{2 \rho n_{1}}{\#\left\{k \in \mathbb{I}: n_{0}<k \leq n_{1}\right\}}<\frac{2 \rho n_{1}}{\beta n_{1} / 4}=\frac{8 \rho}{\beta}<\epsilon .
\end{aligned}
$$

Concerning then the stability of the average sex ratio for historically adapted populations, one obtains an immediate consequence, namely:

Corollary 9. Let $A=\left(a_{1}, a_{2}\right) \in \mathbb{R}^{2}$ be a point of differentiability of $\Gamma_{C}$, and let $\omega^{A}=\left(\begin{array}{l}x_{i}^{A} \\ y_{i}^{A}\end{array}\right)_{i \in \mathbb{N}}$ be an adapted history for the maintenance cost function $C_{A}$. Then, for all $\epsilon>0$ and $M>0$, there exist infinitely many finite histories $\omega^{A}[m, n]=\left(\begin{array}{l}x_{i}^{A} \\ y_{i}^{A}\end{array}\right)_{m \leq i \leq n}$, with $n-m \geq M$, such that

$$
\left|\sum_{k=m+1}^{n}\left(\frac{y_{k}^{A}}{x_{k}^{A}+y_{k}^{A}}-\left(1-f_{C}^{\prime}\left(a_{1}-a_{2}\right)\right)\right)\right|=\left|\sum_{k=m+1}^{n}\left(\frac{x_{k}^{A}}{x_{k}^{A}+y_{k}^{A}}-f_{C}^{\prime}\left(a_{1}-a_{2}\right)\right)\right|<\epsilon .
$$

Proof. Just apply the previous lemma to $\alpha_{i}=\frac{x_{i}^{A}}{x_{i}^{A}+y_{i}^{A}}$ and $\mathbb{I}=\{\lceil M\rceil, 2\lceil M\rceil, 3\lceil M\rceil, \ldots\}$, where $\lceil M\rceil$ denotes the smallest integer greater than or equal to $M$.

We recall that the one-sided derivatives of $f_{C}$ at a point $\Delta$ are given by

$$
f_{C}^{\prime}(\Delta+):=\lim _{H \rightarrow 0^{+}} \frac{f_{C}(\Delta+H)-f_{C}(\Delta)}{H} \quad \text { and } \quad f_{C}^{\prime}(\Delta-):=\lim _{H \rightarrow 0^{-}} \frac{f_{C}(\Delta+H)-f_{C}(\Delta)}{H} .
$$

Since $f_{C}$ is a real valued concave function, its one-sided derivatives are defined everywhere. The next theorem uses the one-sided derivatives of $f_{C}$ to find an estimate for the asymptotic average sex ratio even when it does not converge. 
Theorem 10. Given $B=(b, b-\Delta) \in \mathbb{R}^{2}$, define $L_{\Delta}:=f_{C}^{\prime}(\Delta+)$ and $R_{\Delta}:=f_{C}^{\prime}(\Delta-)$. Then, for all adapted history $\omega^{B}=\left(\begin{array}{l}x_{i}^{B} \\ y_{i}^{B}\end{array}\right)_{i \in \mathbb{N}} \in \Omega$ with respect to $C_{B}$, one has

$$
L_{\Delta} \leq \liminf _{n \rightarrow \infty} \frac{1}{n} \sum_{k=1}^{n} \frac{x_{k}^{B}}{x_{k}^{B}+y_{k}^{B}} \leq \limsup _{n \rightarrow \infty} \frac{1}{n} \sum_{k=1}^{n} \frac{x_{k}^{B}}{x_{k}^{B}+y_{k}^{B}} \leq R_{\Delta} .
$$

Proof. We will prove the result only for the point $B=(0,0)$, since the proof for any other point is analogous. Let $\omega=\left(\begin{array}{l}x_{i} \\ y_{i}\end{array}\right)_{i \in \mathbb{N}} \in \Omega$ be an adapted history with respect to the cost function $C$. For any point $A=(a, a-H)$ with $H>0$, we clearly have

$$
\frac{1}{n} \sum_{k=1}^{n} C_{A}\left(\left(\begin{array}{l}
x_{k-1} \\
y_{k-1}
\end{array}\right),\left(\begin{array}{l}
x_{k} \\
y_{k}
\end{array}\right)\right)=\frac{1}{n} \sum_{k=1}^{n} C\left(\left(\begin{array}{l}
x_{k-1} \\
y_{k-1}
\end{array}\right),\left(\begin{array}{l}
x_{k} \\
y_{k}
\end{array}\right)\right)+\frac{H}{n} \sum_{k=1}^{n} \frac{x_{k}}{x_{k}+y_{k}}+a-H .
$$

Since $\liminf \operatorname{in}_{n \rightarrow \infty}(1 / n) \sum_{k=1}^{n} C_{A}\left(\left(\begin{array}{l}x_{k-1} \\ y_{k-1}\end{array}\right),\left(\begin{array}{l}x_{k} \\ y_{k}\end{array}\right)\right) \geq \Gamma_{C}(A)$ and $\lim _{n \rightarrow \infty}(1 / n) \sum_{k=1}^{n} C\left(\left(\begin{array}{l}x_{k-1} \\ y_{k-1}\end{array}\right),\left(\begin{array}{l}x_{k} \\ y_{k}\end{array}\right)\right)=$ $\Gamma_{C}(0,0)=\gamma$, we obtain

$$
\Gamma_{C}(A) \leq \gamma+H \liminf _{n \rightarrow \infty} \frac{1}{n} \sum_{k=1}^{n} \frac{x_{k}}{x_{k}+y_{k}}+a-H
$$

Therefore, as $f_{C}(H)=\Gamma_{C}(A)-\gamma-(a-H)$, we get that

$$
\frac{f_{C}(H)-f_{C}(0)}{H}=\frac{f_{C}(H)}{H} \leq \liminf _{n \rightarrow \infty} \frac{1}{n} \sum_{k=1}^{n} \frac{x_{k}}{x_{k}+y_{k}}
$$

which yields

$$
f_{C}^{\prime}(0+) \leq \liminf _{n \rightarrow \infty} \frac{1}{n} \sum_{k=1}^{n} \frac{x_{k}}{x_{k}+y_{k}} .
$$

One obtains the inequality $\lim \sup _{n \rightarrow \infty}(1 / n) \sum_{k=1}^{n} x_{k} /\left(x_{k}+y_{k}\right) \leq f_{C}^{\prime}(0-)$ in a similar way, using points $A=(a, a-H)$ with $H<0$.

Remark 11. If $B$ is a point of differentiability of $\Gamma_{C}$, then the left-sided and right-sided derivatives coincide and we clearly recuperate the statement of Theorem 7 . Notice also that, since $f_{C}$ is concave, then both maps $\Delta \mapsto f_{C}^{\prime}(\Delta+)$ and $\Delta \mapsto f_{C}^{\prime}(\Delta-)$ are non-increasing functions and verify $0 \leq f_{C}^{\prime}(\Delta+) \leq f_{C}^{\prime}(\Delta-) \leq 1$ for all $\Delta \in \mathbb{R}$. For any points $B=(b, b-\Delta)$ and $\bar{B}=(\beta, \beta-\bar{\Delta})$, with $\Delta<\bar{\Delta}$, it follows that

$$
R_{\bar{\Delta}}=f_{C}^{\prime}(\bar{\Delta}+) \leq f_{C}^{\prime}(\Delta-)=L_{\Delta},
$$

which implies that the respective intervals $\left[L_{\Delta}, R_{\Delta}\right]$ and $\left[L_{\bar{\Delta}}, R_{\bar{\Delta}}\right]$ may intersect each other only at their common boundary.

Acknowledgement. The authors thank both mathematics departments of UNICAMP and UFSC for the hospitality during the preparation of this manuscript, and their graduate programs for the financial support. M. Sobottka was supported by CNPq-Brazil grant 304457/2009-4 and FUNPESQUISA/UFSC 2009.0138. 


\section{References}

[1] D. Allainé, F. Brondex, L. Graziani, J. Coulon and I. Till-Bottraud, Male-biased sex ratio in litters of Alpine marmots supports the helper repayment hypothesis, Behavioral Ecology 11 (2000), 507-514.

[2] S. Aubry and P. Y. Le Daeron, The discrete Frenkel-Kontorova model and its extensions: I. Exact results for the ground-states, Physica D 8 (1983), 381-422.

[3] V. Bangert, Mather sets for twist maps and geodesics on tori, Dynamics Reported 1 (1988), 1-56.

[4] E. L. Charnov, The theory of sex allocation, Princeton University Press, Princeton, New Jersey, 1982.

[5] C. Darwin, The descent of man, and selection in relation to sex, 2d edition, John Murray, London, 1874.

[6] A. Fathi, Théorème KAM faible et théorie de Mather sur les systèmes lagrangiens, Comptes Rendus des Séances de l'Académie des Sciences, Série I, Mathématique 324 (1997), 1043-1046.

[7] R. A. Fisher, The genetical theory of natural selection, Claredon Press, Oxford, 1930.

[8] D. A. Gomes, Viscosity solution methods and the discrete Aubry-Mather problem, Discrete and Continuous Dynamical Systems, Series A 13 (2005), 103-116.

[9] W. D. Hamilton, Extraordinary sex ratios, Science 156 (1967), 477-488.

[10] J. Hofbauer and K. Sigmund, Evolutionary game dynamics, Bulletin of the American Mathematical Society 40 (2003), 479-519.

[11] J. Komdeur, Facultative sex ratio bias in the offspring of Seychelles warblers, Proceedings: Biological Sciences 263 (1996), 661-666.

[12] G. Szabó and G. Fáth, Evolutionary games on graphs, Physics Reports 446 (2007), 97-216.

[13] R. L. Trivers and D. E. Willard, Natural selection of parental ability to vary the sex ratio of offspring, Science 179 (1973), 90-92.

[14] F. Vollrath, Eusociality and extraordinary sex ratios in the spider Anelosimus eximius (Aranea: Theridiidae), Behavioral Ecology and Sociobiology 18 (1986), 283-287. 